\title{
Polynesia. The Mark and Carolyn Blackburn Collection of Polynesian Art d'Adrienne L. Kaeppler
}

\section{Gilles Bounoure}

\section{(2) OpenEdition \\ 1 Journals}

\section{Édition électronique}

URL : http://journals.openedition.org/jso/6681

DOI : $10.4000 /$ jso.6681

ISSN : $1760-7256$

\section{Éditeur}

Société des océanistes

\section{Édition imprimée}

Date de publication : 30 juin 2012

Pagination : 164-166

ISBN : 978-2-85430-032-1

ISSN : 0300-953x

Référence électronique

Gilles Bounoure, «Polynesia. The Mark and Carolyn Blackburn Collection of Polynesian Art d'Adrienne L. Kaeppler », Journal de la Société des Océanistes [En ligne], 134 | 1er semestre 2012, mis en ligne le 29 juin 2012, consulté le 22 septembre 2020. URL : http://journals.openedition.org/jso/6681 ; DOI : https://doi.org/10.4000/jso.6681

Ce document a été généré automatiquement le 22 septembre 2020.

(c) Tous droits réservés 


\title{
Polynesia. The Mark and Carolyn Blackburn Collection of Polynesian Art d'Adrienne L. Kaeppler
}

\author{
Gilles Bounoure
}

\section{RÉFÉRENCE}

KAEPPLER Adrienne L., 2010. Polynesia. The Mark and Carolyn Blackburn Collection of

Polynesian Art, Honolulu, University of Hawai'i Press (distributed by), xxxvi-412 p., carte, glossaire, bibliogr., index des collectionneurs, 695 objets reproduits, très nombreuses autres illustrations en couleur.

1 Par son ampleur, la qualité de ses reproductions et son ambition, ce livre n'est pas sans évoquer la volumineuse publication consacrée à la «collection Jolika» par son créateur, John Friede, appuyé par plusieurs spécialistes renommés (Friede et al., 2005 ; voir JSO 122-123), et qui offrait la description de 598 objets représentatifs de la plupart des cultures de la Nouvelle-Guinée. Le présent ouvrage est également dévolu à une collection privée de première importance, comparable souvent à celles des plus grands musées, et conçue en sorte de réunir des échantillons de tous les arts traditionnels polynésiens, à l'exception de ceux de Tuvalu, absents de nombreux musées également, ainsi que le relève A. L. Kaeppler dans sa préface (p. xxxiII). L'intérêt de ces objets, pris ensemble ou séparément, suffit à expliquer que cette spécialiste hors pair, aujourd'hui à la tête des collections océaniennes de la Smithsonian Institution, se soit attachée à les publier sous la forme d'un catalogue, qu'elle a fait précéder d'une introduction (pp.1-39) et d'«Islands Essays» (pp. 40-207) consacrés à chacune des cultures polynésiennes représentées.

2 Le nom de M. Blackburn est bien connu à la fois des connaisseurs des arts du Pacifique et des observateurs ou acteurs du marché de l'art, notamment par les publicités illustrées d'objets signalant dans les journaux spécialisés les Mauna Kea Galleries, le 
magasin d'antiquités qu'il tient depuis plusieurs décennies à Honolulu. Il doit à de précoces succès commerciaux dans la numismatique, puis dans le secteur des tapis, de s'être doté rapidement des moyens de compter parmi les grands antiquaires des ÉtatsUnis et du Pacifique, venant également faire affaire en Europe. S'intéressant à titre mimarchand mi-personnel à de nombreux domaines, il a rassemblé maintes collections spécialisées, par exemple sur le thème du surf ou de la mode hawaiienne moderne, le conduisant à publier des livres à leur sujet. De l'impressionnante documentation matérielle, iconographique et livresque qu'il a accumulée, il a tiré un ouvrage consistant et utile sur les traditions du tatouage en Polynésie (Blackburn, 1999), et les quelque quarante mille photographies ou cartes postales à caractère polynésien qu'il a réunies (dont plus de 10000 antérieures à 1900), actuellement en cours d'étude à l'University of East Anglia, devraient donner lieu à une thèse, et, faut-il souhaiter, à d'autres publications. Tandis que se formait le projet de ce livre-catalogue, il confiait à une journaliste (Nicholson, 2007) son souhait de voir sa collection d'objets rejoindre un grand musée, soit le Metropolitan Museum de New York, soit une institution néozélandaise, plus facilement accessible aux visiteurs polynésiens.

3 Comme le signale A. L. Kaeppler p. 412, les chapitres régionaux qu'elle a placés à la suite de son introduction présentant l'ensemble de la Polynésie et de ses arts du point de vue historique et typologique reprennent largement les exposés qu'elle avait publiés dans Art Océanien (1993, pp. 519-546). Elle les a parfois abrégés (ainsi des pages qu'elle consacre aux îles Marquises, pp. 95 sq., qui ne mentionnent pas les recherches de Von den Steinen, Linton, E. S. C. et Willowdean Handy et Carol Ivory auxquelles renvoyait son texte de 1993, p. 531). Elle les a plus souvent augmentés, pour citer des objets que ne mentionnait pas son texte primitif (ainsi des pièces de tapa associées aux crânes trophées des Marquises, pp. 101-105 ou des herminettes à lames en coquillage de Nive, p. 87, dont ne faisaient pas état les paragraphes correspondants de 1993, pp. 532-3 et 529). Elle en a légèrement modifié la succession, le volume de 2010 se fermant sur Hawaii, tandis que l'exposé de 1993 concluait, selon un ordre plus strictement chronologique, par la Nouvelle-Zélande, avec un alinéa sur les îles Chatham qui manque ici, bien que le catalogue qui suit décrive et reproduise une très rare massue en schiste Moriori ( $n^{\circ} 486$ p. 347).

Ce catalogue doit son intérêt à la fois à la qualité des objets reproduits, à l'excellence de leurs reproductions, et à l'expertise des notices très concises dont les a accompagnés $\mathrm{A}$. L. Kaeppler. Le nombre de pièces de grande ancienneté ou d'extrême rareté qu'a su saisir l'antiquaire collectionneur sur le marché de l'art a de quoi frapper aussi bien les initiés que les profanes. La grande exposition consacrée aux collectes de Cook par A. L. Kaeppler en 2008-2010 avait été l'occasion de citer dans cette rubrique (Jso 130-131), parmi les fleurons de la collection Blackburn, la massue (ou 'Akau tau) historiée de 112 gravures figuratives que l'explorateur avait collectée à Tonga lors de son troisième voyage, et qu'il tenait probablement du Tu 'i Tonga lui-même; on en trouvera une description et des vues plus détaillées dans cet ouvrage ( $n^{\circ} 169$, p. 252, voir aussi pp. 66-67). Dans la même livraison du JSO, une évocation des arts de Takuu rappelait les malheurs successifs de ses insulaires et le nombre très réduit d'objets témoignant aujourd'hui de leur ancienne civilisation; la "figure d'ancêtre » de Takuu ( $\mathrm{n}^{\circ} 225$, pp. 271 et 92) de la collection Blackburn paraît tout aussi précieuse que ses trois homologues précédemment connus, conservés au musée de Leipzig. Pour clore ces remarques qu'on pourrait étendre à plusieurs dizaines de pièces de cette collection, un 
bol à kava de Futuna ( $n^{\circ} 211$, pp. 266 et 82), orné au revers de motifs peints analogues à ceux des tapas locaux, y voisine avec l'un des plus anciens salatasi qu'on connaisse de cette île ( ${ }^{\circ} 208$, p. 265), collecté par Abel Du Petit-Thouars aux alentours de 1840.

Sous ce dernier nom, l'index des collecteurs et collectionneurs figurant en fin d'ouvrage recense 18 autres objets, tous originaires des îles Marquises et encore dans la famille de l'amiral quand Paul et Francine De Dekker les exposèrent et les publièrent en 1982. Du Petit-Thouars les aurait reçus en 1843 du chef Iotete, lors de sa prise de possession de l'archipel au nom de la France. Mais ces pièces ne sont pas les plus anciennement collectées aux Marquises, puisqu'un étrier d'échasse (tapuvae, n 290 p. 289) orné de deux tikis superposés, figuration peu fréquente et absente des étriers plus récents, se trouve formellement identifié par A. L. Kaeppler comme celui que le commander David Porter avait rapporté de son occupation de l'archipel au nom des États-Unis (1813-1814) et reproduit dans sa relation de voyage. Eric Kjellgren (2005, pp. 17 et $\left.96, n^{\circ} 66\right)$ avait déjà esquissé ce rapprochement mais sans s'avancer au-delà des similitudes constatées. Certes, les 84 objets constituant la partie marquisienne de cette collection ne couvrent pas toutes les traditions artistiques de cet archipel, mais ils en fournissent d'excellents témoins, et le parcours des autres sections de l'ouvrage fait aboutir au même type de conclusion.

On relèvera pour finir que ni A. L. Kaeppler ni M. Blackburn n'ont éprouvé le besoin de recourir aux techniques modernes d'exploration et d'identification physico-chimiques de plus en plus utilisées par d'autres collectionneurs ou spécialistes d'art océanien, y compris pour des objets d'ancienneté et de matériau comparables. Pour les pièces difficiles à dater (tels un pendentif maori en pierre possiblement $\mathrm{du} \mathrm{xv}^{\mathrm{e}}$ siècle ou un pilon marquisien excavé dans une tranchée profonde qui le ferait remonter au $\mathrm{xVI}^{\mathrm{e}}$ siècle) comme pour celles se prêtant mieux aux analyses de laboratoire, ils ont préféré s'en tenir aux procédés traditionnels d'expertise fondés sur l'expérience, l'examen visuel et les données historiques disponibles sur les objets, leur collecte et leur circulation en Occident. Telle était déjà la méthode des Beasley, Oldman et Hooper dont ce livre salue aussi les noms et les parcours, en même temps que ceux d'autres collectionneurs marchands entre les mains desquels passèrent maints objets collectés par les équipages de Cook avant d'orner aujourd'hui cette collection. On ne peut que lui souhaiter de venir enrichir les musées néo-zélandais à l'instar de celle qu'avait jadis rassemblée William Oldman, dont les mânes, de la sorte, n'auront pas été invoqués en vain.

\section{BIBLIOGRAPHIE}

BLACKBURN Mark, 1999. Tattoos from Paradise. Traditional Polynesian Patterns, Atglen, Pa., Schiffer Publishing.

FRIEDE John, Gregory HODGINS, Philippe PELTIER, Dirk SMIDT and Robert L.WELSCH, 2005. New Guinea Art, Masterpieces from the Jolika Collection of Marcia and John Friede, San Francisco - Milan, Fine Arts Museums of San Francisco - 5 Continents Éditions, 2 vol. 
KAEPPLER Adrienne L., Christian KAUfMANn et Douglas NEWTON, 1993. Art océanien, Paris, CitadellesMazenod.

KAEPPLER Adrienne L. (ed.), 2009. James Cook and the Exploration of the Pacific, Londres, Thames \& Hudson.

KJELLGREN Eric with Carol S. IVORY, 2005. Adorning the World. Art of the Marquesas Islands, New YorkNew Haven, Metropolitan Museum-Yale University Press.

NICHOLSON Louise, 2007 (octobre). A Rolls-Royce collection of Oceanic art, Apollo Magazine (Londres). 